\title{
Parallel Ion Flow Velocity Measurement Using Laser Induced Fluorescence Method in an Electron Cyclotron Resonance Plasma
}

\author{
Shinji YOSHIMURA, Atsushi OKAMOTO ${ }^{1)}$, Kenichiro TERASAKA ${ }^{2)}$, \\ Kohei OGIWARA $^{2)}$, Mitsutoshi ARAMAKI ${ }^{3)}$ and Masayoshi Y. TANAKA ${ }^{2)}$ \\ National Institute for Fusion Science, Toki 509-5292, Japan \\ ${ }^{1)}$ Department of Quantum Science and Energy Engineering, Tohoku University, Sendai 980-8579, Japan \\ ${ }^{2)}$ Interdisciplinary Graduate School of Engineering Science, Kyushu University, Kasuga 816-8580, Japan \\ ${ }^{3)}$ Department of Electrical Engineering and Computer Science, Nagoya University, Nagoya 464-8603, Japan
}

(Received 21 December 2009 / Accepted 23 February 2010)

Parallel ion flow velocity along a magnetic field has been measured using a laser induced fluorescence (LIF) method in an electron cyclotron resonance (ECR) argon plasma with a weakly-diverging magnetic field. To measure parallel flow velocity in a cylindrical plasma using the LIF method, the laser beam should be injected along device axis; however, the reflection of the incident beam causes interference between the LIF emission of the incident and reflected beams. Here we present a method of quasi-parallel laser injection at a small angle, which utilizes the reflected beam as well as the incident beam to obtain the parallel ion flow velocity. Using this method, we observed an increase in parallel ion flow velocity along the magnetic field. The acceleration mechanism is briefly discussed on the basis of the ion fluid model.

(c) 2010 The Japan Society of Plasma Science and Nuclear Fusion Research

Keywords: ion flow, flow velocity measurement, laser induced fluorescence, tunable dye laser, metastable argon ion, diverging magnetic field, ion acceleration, ECR plasma

DOI: $10.1585 /$ pfr.5.S2052

\section{Introduction}

Acceleration of charged particles along a magnetic field line has been studied extensively in various fields of plasma research in relation to a variety of astrophysical and magnetospheric phenomena and the development of electric thrusters based on plasma propulsion. In particular, ion acceleration in plasmas with converging and diverging magnetic fields has been a topic of interest; a number of laboratory experiments have been performed in recent years [1-4] in which flow velocity measurement is an essential element.

Directional Langmuir probes (DLPs) [5] or Mach probes that provide an easy-to-use way to measure ion flow velocity profiles have been widely used in preceding experiments. The ion Mach number, which is defined by the ratio of the ion flow velocity to the ion sound speed, can be determined by applying a certain theoretical model. Note that the obtained ion Mach number differs nonnegligibly depending on the model used for analysis. On the other hand, the laser induced fluorescence (LIF) method [6] has been regarded as a powerful tool for flow velocity measurement, since the absolute value can be directly obtained from the Doppler shift of the LIF spectrum. We have already reported the results of azimuthal and radial ion flow velocity measurements using the LIF method in the HYPER-I device at the National Institute for Fusion Science $[7,8]$; however, the parallel ion flow velocity has not

author'se-mail: yshinji@nifs.ac.jp been measured yet due to difficulties arising from the geometrical limitations of the experiment and the lack of a wavelength standard.

In this paper, we present the initial results of parallel ion flow velocity measurement using the LIF method in the HYPER-I device, i.e., in an ECR plasma with a weakly-diverging magnetic field. In the sections that follow, we first describe the experimental setup and the LIF measurement technique employed in this study. The experimental results, including the separation of LIF emission induced by the incident and reflected beams and an observed increase in ion flow velocity along the magnetic field, are then shown; the acceleration mechanism is discussed briefly. Concluding remarks are given in the last section.

\section{Experimental Details}

\subsection{The HYPER-I device}

The experiments were performed in the HYPER-I device. A schematic of the device with the axial profile of the magnetic field strength $(B)$ is shown in Fig. 1 . The HYPER-I device consists of a cylindrical vacuum chamber of $300 \mathrm{~mm}$ in diameter and $2000 \mathrm{~mm}$ in length with ten magnetic coils. The magnetic field configuration is a weakly-diverging one, a so called magnetic beach: $B=$ $0.0912 \mathrm{~T}$ at $z=950 \mathrm{~mm}$, and $B=0.0709 \mathrm{~T}$ at $z=1400 \mathrm{~mm}$, where $z$ denotes the axial distance from the microwave in- 


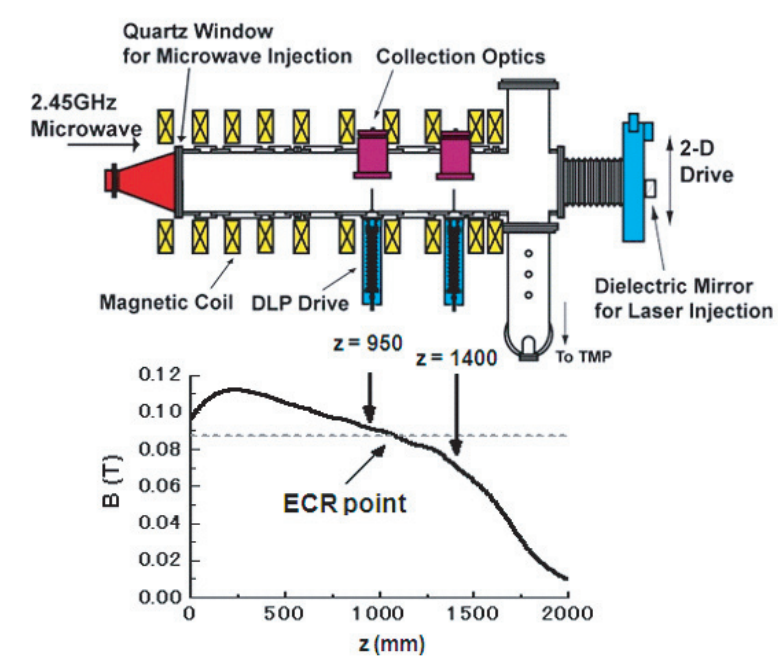

Fig. 1 Schematic of the HYPER-I device with the axial profile of the magnetic field strength. Measurement positions are indicated by arrows.

jection window. Here we use a Cartesian coordinate system whose origin is at the center of the microwave injection window. The $z$-axis is along the axis of the chamber and points toward the end of the chamber. The $x$-axis ( $y$-axis) lies in the horizontal (vertical) plane and points rightward (upward) as viewed from the end of the chamber.

The laser beam was injected along the negative $z$-axis through a quartz window installed at the end of the device. The collection optics for detecting LIF emission were located at $z=950 \mathrm{~mm}$ and $z=1400 \mathrm{~mm}$. The horizontal positions of the collection optics were fixed at $x=0 \mathrm{~mm}$.

Plasmas are produced by an ECR discharge at a frequency of $2.45 \mathrm{GHz}$, in which the resonance condition is fulfilled at $B=0.0875 \mathrm{~T}(z=1080 \mathrm{~mm})$. The microwave input power and the pressure of argon gas were $6 \mathrm{~kW}$ and $8.3 \times 10^{-5}$ Torr, respectively. The typical electron density and electron temperature were $1.5 \times 10^{11} \mathrm{~cm}^{-3}$ and $7 \mathrm{eV}$, respectively.

\subsection{LIF measurement}

We employed a doublet transition scheme that has been routinely used for LIF measurement in argon plasmas with a dye laser [9-13]. In this scheme, the laser wavelength is tuned to $611.49 \mathrm{~nm}$, which is the resonant absorption wavelength of the $3 d^{2} G_{9 / 2}-4 p^{2} F_{7 / 2}$ transition of a metastable argon ion. The $4 p^{2} F_{7 / 2}$ excited state has a very short lifetime and spontaneously decays into the $4 s^{2} D_{5 / 2}$ state by emitting a $460.96 \mathrm{~nm}$ photon, which constitutes the LIF signal. The LIF spectrum can be obtained by tuning the laser wavelength through the transition wavelength of $611.49 \mathrm{~nm}$.

A tunable dye laser pumped by an Nd:YAG laser pulsed at $30 \mathrm{~Hz}$ was used. The laser wavelength was controlled by a PC and monitored with a wavemeter. The laser beam was transferred with mirror optics and injected from the quartz window installed at the end of the de-
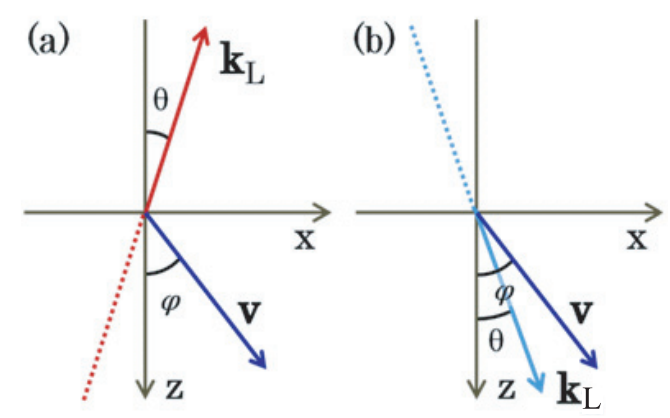

Fig. 2 LIF geometry of (a) incident beam and (b) reflected beam, where the wave vectors and velocity vectors lie in the $x-z$ plane.

vice. The last mirror for laser injection was installed on two-dimensional positioning system, so that we can adjust the laser injection position flexibly. The collection optics consists of a lens, an interference filter, and a photomultiplier tube (PMT). The LIF signal (PMT output) was amplified by a high-speed amplifier and averaged over 100 laser shots for each laser wavelength with a gated integrator. Details of the LIF measurement system have been described in ref. [8].

The LIF method for velocity measurement utilizes the Doppler effect. The laser frequency $v_{\mathrm{L}}$ that is absorbed by a particle moving at velocity $\boldsymbol{V}$ is

$$
2 \pi \Delta v=2 \pi\left(v_{\mathrm{L}}-v_{0}\right)=\boldsymbol{k}_{\mathrm{L}} \cdot \boldsymbol{V},
$$

where $v_{0}$ denotes the resonant absorption frequency in the rest frame, and $\boldsymbol{k}_{\mathrm{L}}$ is the wave vector of the laser beam. Here we consider the case of oblique injection at an angle $\theta$ relative to the $z$-axis in the $x$ - $z$ plane as shown in Fig. 2(a). The wavelength of the incident beam $\lambda_{\mathrm{Li}}(=$ $\left.\mathrm{c} / v_{\mathrm{Li}}=2 \pi / k_{\mathrm{Li}}\right)$ that satisfies Eq. (1) is

$$
\begin{aligned}
& \mathrm{c}\left(\frac{1}{\lambda_{\mathrm{Li}}}-\frac{1}{\lambda_{0}}\right)=\frac{1}{\lambda_{\mathrm{Li}}} V \cos [\pi-(\theta+\varphi)], \\
& \lambda_{\mathrm{Li}}=\lambda_{0}\left[1+\frac{V}{\mathrm{c}} \cos (\theta+\varphi)\right], \text { or } \\
& \lambda_{\mathrm{Li}}=\lambda_{0}\left[1+\frac{V_{z}}{\mathrm{c}} \cos \theta\left(1-\frac{V_{x}}{V_{z}} \tan \theta\right)\right],
\end{aligned}
$$

where $\left(V_{x}, V_{z}\right)=(V \sin \varphi, V \cos \varphi)$. For $\theta=0$, Eq. (4) is reduced to a familiar expression for calculating parallel ion flow velocity $V_{z}$ from the Doppler shift of the LIF spectrum,

$$
V_{z}=\frac{\mathrm{c}\left(\lambda_{\mathrm{Li}}-\lambda_{0}\right)}{\lambda_{0}} .
$$

To determine $V_{z}$ from Eq. (5), the accuracy of the absolute laser wavelength is a critical problem. Generally, the absolute accuracy of a wavemeter (a few pm) is not sufficient to measure ion flow velocity; a wavelength standard such as an iodine cell spectrum is indispensable. When we measure perpendicular flow velocity in a cylindrically symmetric flow field, the peak of the LIF spectrum measured on the $z$-axis can be used as a wavelength standard. However, 
no such symmetry exists in the $z$-direction. Moreover, the condition $\theta=0$ requires that the laser beam be injected into the plasma exactly along the $z$-axis from one end of the device; the incident beam is subjected to reflection by the microwave injection window located at the other end in our experimental configuration. The LIF emission induced by this reflected beam may interfere with that of the incident beam, causing at least an undesirable broadening of the obtained LIF spectrum, or leading to a significant asymmetry in the shape of the LIF spectrum, if unresolved. When we attempt to avoid this interference by making a small angle $\theta$ to the incident beam, a finite perpendicular velocity $V_{x}$ may cause an error, as shown by Eq. (4).

However, we can eliminate this uncertainty by using the reflected beam. As shown in Fig. 2(b), the reflected beam is geometrically symmetric against the incident beam; the wavelength of the reflected beam $\lambda_{\mathrm{Lr}}$ that satisfies Eq. (1) is obtained by changing the argument of the cosine function in Eq. (2) from $\pi-(\theta+\varphi)$ to $\varphi-\theta$, which yields

$$
\lambda_{\mathrm{Lr}}=\lambda_{0}\left[1-\frac{V}{\mathrm{c}} \cos (\theta-\varphi)\right] .
$$

By subtracting Eq. (6) from Eq. (3), we obtain

$$
\lambda_{\mathrm{Li}}-\lambda_{\mathrm{Lr}}=2 \lambda_{0} \frac{V_{z}}{\mathrm{c}} \cos \theta .
$$

For quasi-parallel injection, $\theta \sim 0$, we have

$$
V_{z}=\frac{\mathrm{c}\left(\lambda_{\mathrm{Li}}-\lambda_{\mathrm{Lr}}\right) / 2}{\lambda_{0}} .
$$

In Eq. (8), the Doppler shift of the LIF spectrum appearing in Eq. (5) is replaced by half of the relative shift between two LIF spectra obtained from the incident and reflected beams, which can be measured more precisely than the absolute wavelength measured with a wavemeter. In addition, the mean value of $\lambda_{\mathrm{Li}}$ and $\lambda_{\mathrm{Lr}}$ gives the resonant absorption wavelength in the rest frame, provided that $V_{x} \sim 0$ can be assumed, which is probably valid on the $z$-axis.

$$
\frac{\lambda_{\mathrm{Li}}+\lambda_{\mathrm{Lr}}}{2}=\lambda_{0}\left(1-\frac{V_{x}}{\mathrm{c}} \sin \theta\right) \approx \lambda_{0} \text {. }
$$

This relation can be used for the absolute wavelength calibration of the wavemeter used in this study.

\section{Results and Discussion}

\subsection{Separation of LIF emission}

Figure 3 shows the dependence of LIF intensity on the laser injection position, where the laser wavelength was fixed at $611.492 \mathrm{~nm}$. LIF emission induced by the incident and reflected beams is successfully separated; the incident angle $\theta \sim 10^{-2} \mathrm{rad}$, which can be considered quasi-parallel to the $z$-axis. The spatial resolution of the LIF measurement is about $10 \mathrm{~mm}$.

\subsection{Parallel ion flow velocity}

Figure 4 shows LIF spectra taken at different positions, (a) $z=950 \mathrm{~mm}$ and (b) $z=1400 \mathrm{~mm}$, where the
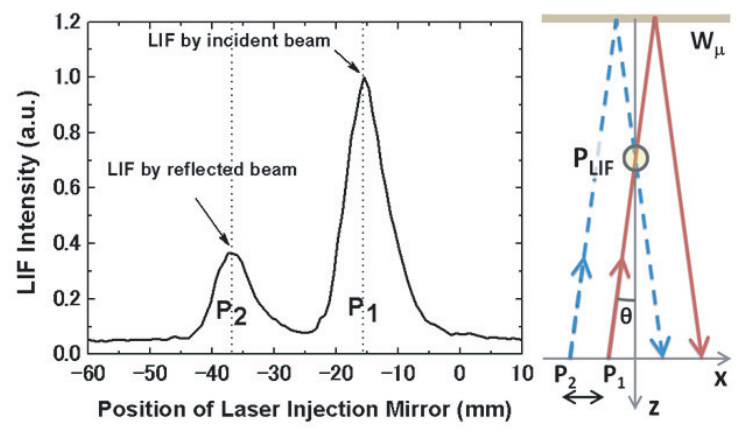

Fig. 3 Intensity of LIF signal measured at $z=950 \mathrm{~mm}$ as a function of laser injection position. Incident angle $\theta$ is depicted in an exaggerated manner. $\mathrm{W}_{\mu}$ is the microwave injection window, $\mathrm{P}_{\mathrm{LIF}}$ is the LIF measurement position, and $\mathrm{P}_{1}$ and $\mathrm{P}_{2}$ are the laser injection positions of the incident and reflected beams, respectively.
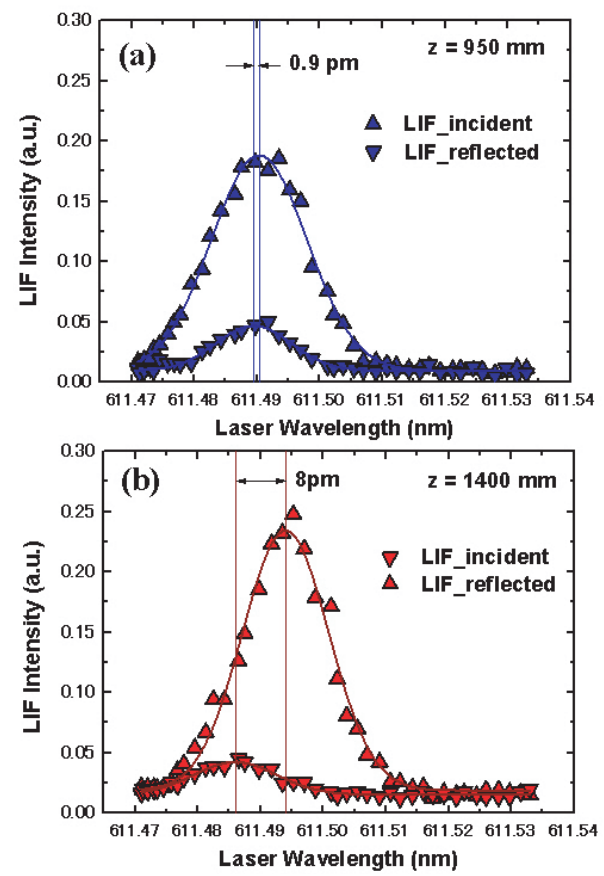

Fig. 4 LIF spectra taken at (a) $z=950 \mathrm{~mm}$ and (b) $z=1400 \mathrm{~mm}$. Parallel ion flow velocities calculated from Eq. (8) are (a) $213 \mathrm{~m} / \mathrm{s}$ and (b) $1965 \mathrm{~m} / \mathrm{s}$.

absolute wavelength on the horizontal axis is calibrated by using Eq. (9). In Fig. 4 (a), the shift between the two LIF spectra obtained by the incident and reflected beams is small, which corresponds to a small parallel ion flow velocity. This result may be attributed to the fact that the measurement position is in the ECR region. On the other hand, a noticeable shift is observed in Fig. 4 (b), indicating a significant increase in parallel ion flow velocity along the weakly-diverging magnetic field.

The results, including the observed shifts, the flow velocities calculated from Eq. (8), and the ion Mach numbers, are listed in Table 1. Although parallel acceleration of ions is clearly visible in Table 1 , the velocity is still in 
Table 1 Parallel ion flow velocities calculated from the relative shifts $\Delta \lambda$ of two LIF spectra obtained by the incident and reflected beams. $V_{z} / \mathrm{C}_{\mathrm{s}}$ is the ion Mach number.

\begin{tabular}{c|c|c|c}
\hline$z(\mathrm{~mm})$ & $\Delta \lambda(\mathrm{pm})$ & $V_{z}(\mathrm{~m} / \mathrm{s})$ & $V_{z} / \mathrm{C}_{\mathrm{s}}$ \\
\hline 950 & 0.87 & 213 & 0.052 \\
1400 & 8.02 & 1965 & 0.480 \\
\hline
\end{tabular}

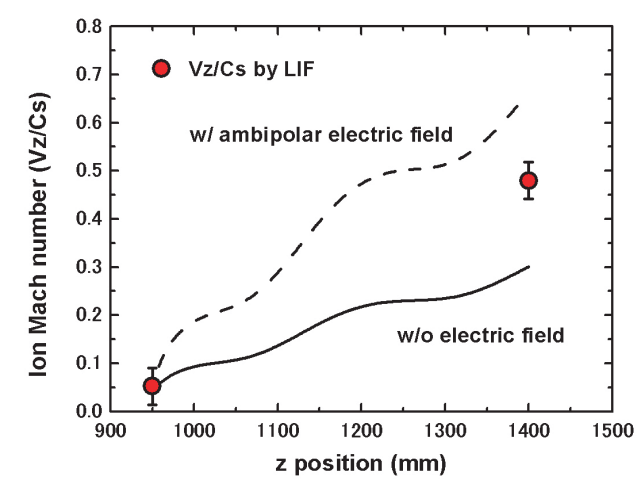

Fig. 5 Increase in parallel ion flow velocity along the magnetic field in terms of ion Mach number. Solid line shows expected acceleration under the assumption of no axial electric field. Broken line shows acceleration when an ambipolar electric field is present. Error bars are evaluated from the precision of curve fitting and stability of the wavemeter.

the subsonic regime at $z=1400 \mathrm{~mm}$. A supersonic ion flow might be expected in downstream regions where the magnetic field is more strongly divergent.

\subsection{Ion acceleration mechanism in parallel direction}

Here we briefly discuss the ion acceleration mechanism along the weakly-diverging magnetic field. The onedimensional Bernoulli's equation is derived from the ion fluid equation without an electric field term,

$$
\frac{M^{2}}{2}+\kappa_{1} \ln n+\left(1-\kappa_{1}\right) \Psi=\text { const. }
$$

where $M$ is the ion Mach number, $\kappa_{1}=T_{\mathrm{i}} /\left(T_{\mathrm{e}}+T_{\mathrm{i}}\right)$, $\Psi \equiv \mu_{\mathrm{i}} B / k_{\mathrm{B}} T_{\mathrm{e}}, \mu_{\mathrm{i}}$ is the magnetic moment, and $k_{\mathrm{B}}$ is the Boltzmann constant. The second and third terms on the left-hand side are related to the ion thermal pressure and to inhomogeneity of the magnetic field, respectively. Detailed discussion based on this expression as well as the derivation of Eq. (10) is beyond the scope of this paper and will be published elsewhere. Using Eq. (10) and assuming strongly magnetized electrons, the expected acceleration is shown as a solid line in Fig. 5; it is not sufficient to explain the experimental results. However, the acceleration seems to be overestimated with the assumption of an ambipolar electric field (broken line in Fig. 5). A detailed examination of the ion acceleration mechanism will be our future work.

\section{Conclusion}

Parallel ion flow velocity has been measured using the LIF method in an ECR argon plasma with a weaklydiverging magnetic field. To measure parallel flow velocity in a cylindrical plasma, a laser beam should be injected along device axis from one end; however, the incident beam is reflected at the other end when a beam damp cannot be installed appropriately. To avoid interference between the LIF emission induced by the incident and reflected beams, we used a small angle with respect to the incident beam. This quasi-parallel injection enables us to distinguish the LIF spectrum obtained from the incident beam from that of the reflected beam, and to determine the absolute ion flow velocity from half of the shift between those LIF spectra. In addition, the mean value of the center wavelengths of those LIF spectra gives the resonant absorption wavelength of metastable ions in rest frame, which can be used as a wavelength standard for calibrating a wavemeter. Using this method, we observed an increase in parallel ion flow velocity along the magnetic field up to the ion Mach number of 0.48 . This acceleration cannot be explained without the existence of an axial electric field. Parallel ion flow velocity measurements in more strongly divergent regions, where a supersonic ion flow might be expected, will be our future work.

\section{Acknowledgments}

This work was supported in part by the NIFS Collaboration Research Program (NIFS08KQHP012).

[1] H. Tobari, A. Ando, M. Inutake and K. Hattori, Phys. Plasmas 14, 093507 (2007).

[2] A. Ando, T. Hagiwara, T. Komagome, K. Hattori and M. Inutake, Plasma Fusion Res. 3, S1018 (2008).

[3] K. Yoshida, T. Shibata, A. Nezu, H. Matsuura and H. Akatsuka, J. Plasma Fusion Res. SERIES 8, 923 (2009).

[4] C. A. Deline, R. D. Bengtson, B. N. Brieman, M. R. Tushentsov, J. E. Jones, D. G. Chavers, C. C. Dobson and B. M. Schuettpelz, Phys. Plasmas 16, 033502 (2009).

[5] K. Nagaoka, A. Okamoto, S. Yoshimura and M. Y. Tanaka, J. Phys. Soc. Jpn. 70, 131 (2001).

[6] R. A. Stern and J. A. Johnson III, Phys. Rev. Lett. 34, 1548 (1975).

[7] A. Okamoto, S. Yoshimura, S. Kado and M. Y. Tanaka, J. Plasma Fusion Res. 80, 1003 (2004).

[8] S. Yoshimura, A. Okamoto and M. Y. Tanaka, J. Plasma Fusion Res. SERIES 8, 11 (2009).

[9] W. Gräfen, M. v. Hoesslin, H. Kempkens and J. Uhlenbusch, Phys. Fluids 31, 3044 (1988).

[10] N. Sadeghi, T. Nakano, D. J. Trevor and R. A. Gottscho, J. Appl. Phys. 70, 2552 (1991).

[11] M . J . Goeckner, J. Goree and T. E. Sheridan, Phys. Fluids B 3, 2913 (1991).

[12] D. A. Edrich, R. McWilliams and N. S. Wolf, Rev. Sci. Instrum. 67, 2812 (1996).

[13] I. A. Biloiu, E. E Scime and C. Biloiu, Plasma Sources Sci. Technol. 18, 025012 (2009). 